\title{
Physical Layer Security in Multiuser VLC Systems with a Randomly Located Eavesdropper
}

\author{
Sunghwan Cho*, Gaojie Chen", and Justin P. Coon* \\ *Department of Engineering Science, University of Oxford, OX1 3PJ, United Kingdom. \\ \{sunghwan.cho and justin.coon\}@eng.ox.ac.uk \\ ${ }^{\#}$ Department of Engineering, University of Leicester, LE1 7RH, United Kingdom. \\ gaojie.chen@leicester.ac.uk
}

\begin{abstract}
This paper proposes a secrecy enhancement mechanism for multiuser visible light communication (VLC) systems. Thanks to the inherent advantages of visible light that it cannot penetrate opaque walls and its channel gain largely depends on the distance, VLC systems can serve multiple users at a time with high security and dense spatial reuse. Nevertheless, in the presence of multiple users, the interference caused by other users' signals should be carefully considered when analyzing the secrecy rate and data rate performance measures. By employing a continuous LED model, we formulate an optimization problem to find the optimal set of LEDs that should be used for communication such that the average secrecy rate for the secured user is maximized while satisfying data rate requirements for the other ordinary users. Numerical results are provided to verify that the relative locations of multiple users, the spatial distribution of a random eavesdropper, and the required data rates are significant factors that affect the secrecy performance in multiuser VLC systems.
\end{abstract}

Index Terms-Physical layer security, visible light communication, multiuser, stochastic geometry, secrecy rate.

\section{INTRODUCTION}

Visible light communication (VLC) has been regarded as a promising solution to resolve the shortfall of RF spectrum by using the visible light spectrum for wireless communications. Because visible light cannot penetrate opaque walls and due to the fact that the VLC channel largely depends on the distance between a transmitter and a receiver, VLC systems can provide high security and dense spatial reuse. Therefore, it is expected that VLC serve numerous users simultaneously with high data rates and short time delays [1].

Also VLC provides high security to some degree, the possibility that an eavesdropper (ED) can wiretap the transmitted information in an open area still exists. Notably, in multiuser VLC systems in large open spaces - such as libraries, offices, and shopping malls - it would be challenging to distinguish between legitimate and malicious users. Therefore, various physical layer security (PLS) techniques have been proposed and studied to secure the transmission in VLC systems. PLS is a set of methods that utilize the channel randomness to transmit information securely at the cost of reducing the communication rate [2].

In the recent literature, many PLS studies for VLC systems have been reported [3]-[5]. However, the majority of works have focused on single-user VLC systems. Although [6]-[8] proposed PLS techniques for multiuser VLC systems using various transmission schemes, such as zero-forcing transmission, artificial-noise-aided precoding, and non-orthogonal multiple access (NOMA), these studies have limitations with regard to performance and feasibility. More specifically, in [6], Pham et al employed a zero-forcing precoder to maximize the secrecy sum-rate, however, when the channel state information (CSI) of the ED is not available, the proposed scheme behaves only to increase the achievable sum-rate without taking the ED's reception into account; thus, this approach can be highly susceptible to eavesdropping depending on the relative locations of the UEs and ED. Also, in [7], Arfaoui et al designed a precoding matrix that improves various secrecy measures, but assumed that all the UEs are active and their CSI is known to the LEDs, which may not be practical in some cases. On the other hand, in [8], Zhao et al investigated the secrecy outage probability (SOP) for multiuser VLC systems with NOMA. However, the model was limited in that it assumed only one LED transmitter, which would not be practical for multiuser VLC systems implemented in large open spaces. Note that, in reality, many LEDs are widely distributed to evenly illuminate an expansive area in accordance with lighting requirements.

In our recent work [4], we proposed a VLC multipleinput single-output (MISO) beamforming scheme that can be implemented without the knowledge of an ED's precise location, utilizing its statistical information instead. Only one UE was considered in the downlink in that work. We showed that the beamforming scheme could be well approximated by an LED selection scheme, where the nearest LED from the UE is selected to transmit important data to the UE. Compared to beamforming, LED selection has advantages regarding low computational complexity and simple implementation [4].

Inspired by the simple LED selection scheme, in this paper, we propose an LED selection technique for multiuser VLC systems that requires the statistical information of the ED's random location. Unlike LED selection in the single-user VLC system, where only the nearest LED is chosen to transmit, selecting a proper number of LED transmitters is necessary for multiuser VLC scenarios. More specifically, if too many LEDs are selected to transmit information signals, the signals would not only reach the intended UE but also cause severe interference to other users. Also, from the secrecy perspective, transmission from the excessive number of LEDs facilitates eavesdropping, which would degrade the secrecy performance. 


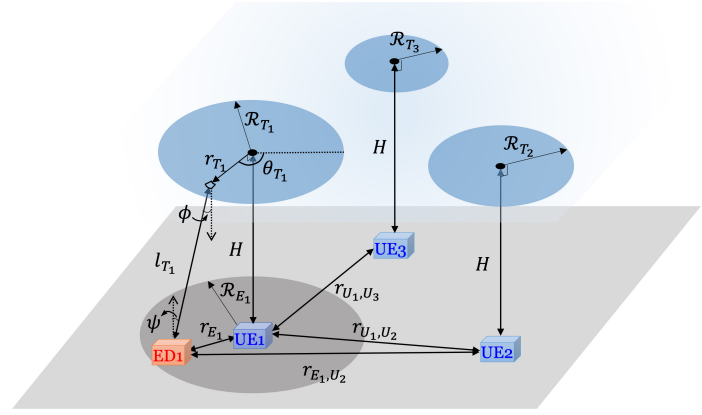

(a) Geometry with multiple UEs and one ED.

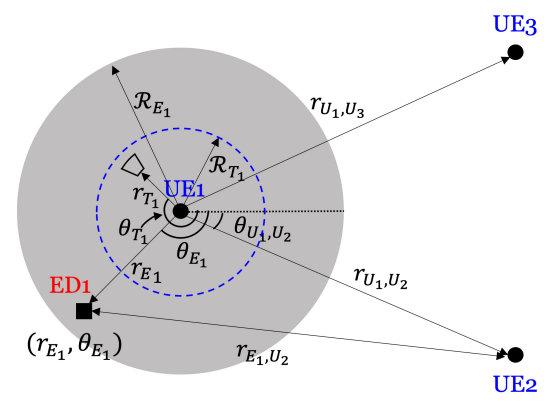

(b) Geometry projected on the work plane

Fig. 1. A VLC system with a continuous LED model.

In contrast, if too few LEDs are selected, the transmitted signals may be too weak to reach the intended user. Therefore, we devise a method to determine the optimal set of LEDs that transmit information signals for maximizing the average secrecy rate for the secured user, while satisfying the required data rates for the ordinary users. An optimization problem to choose the best set of LEDs is formulated by utilizing a continuous LED model, which can mitigate complications that arise in the mathematical formulation of the problem. Unlike the beamforming schemes reported in [3]-[7] where all the LEDs in the room participate in producing a beamsteering strategy, the proposed LED selection scheme requires the activation of only the LEDs located near to the UEs. This trait would improve the spatial efficiency of VLC systems and permit a more straightforward implementation in terms of low synchronization requirement and computational complexity.

\section{System ModeL}

\section{A. Room Configuration with Continuous LEDs}

Throughout the paper, as shown in Fig. 1, we consider a multiuser VLC network consisting of $N$ UEs, which are denoted by $\mathrm{UE}_{i}$ for $i \in\{1,2, \ldots, N\}$. Each UE is assumed to be threatened by a corresponding ED, which tries to wiretap the transmitted information signal from the LEDs to the targeted UE. Also, we assume that only one UE utilizes the secured transmission using PLS techniques at a time; thus there is only one ED of interest at a time. This assumption can be justified since a PLS technique may be a means to refresh the secret keys used in upper layers, rather than to exchange a continuous large data stream; the number of concurrent users that require the secured PLS transmission can be limited to one. However, note that every UE can request a secured PLS transmission to LEDs in a time-slotted manner. All receivers are assumed to be located on the same gray work plane as shown in Fig. 1(a).

As shown in Fig. $1(\mathrm{~b}), \mathrm{ED}_{i}(i=1$ in the figure) is randomly located around $\mathrm{UE}_{i}$. In other words, $\mathrm{ED}_{i}$ is located at $\left(r_{E_{i}}, \theta_{E_{i}}\right)$, whose origin is the location of $\mathrm{UE}_{i}$, and $r_{E_{i}}$ $\left(r_{E_{i}} \leq \mathcal{R}_{E_{i}}\right)$ and $\theta_{E_{i}}\left(0 \leq \theta_{E_{i}}<2 \pi\right)$ are random variables. Since the channel gain in VLC largely depends on the distance between an LED and a receiver's photodiode (PD) [9], it can be justified that the ED tries to be located near to the UE.

In addition, rather than considering discrete LED transmitters as is the case in most of the related recent literature [3][8], in this paper, we consider a continuous LED model as illustrated in Fig. 1(a), where an infinite number of LED transmitters are attached to the ceiling of an infinitely large room, and the distances between the LEDs are infinitesimal. As shown in [10], this continuum model significantly simplifies the calculation of the received optical power, while accurately approximating the real LED transmitters, i.e., the discrete model. Moreover, the continuum model can be transferred to the discrete LED model with a low complexity by utilizing the selection scheme minimizing the sum of the distances between the continuous and discrete transmitters [10]. Note that, in reality, an LED fixture consisting of multiple individual LEDs needs to be capable of communicating independently of other fixtures to serve multiple users at a time [4]. Similarly, in this paper, any subset of a continuous LED plane is assumed to act as an independent access point. By assuming that the LEDs retain knowledge of the locations of the UEs, we assume that the information transmitters are located right above each $\mathrm{UE}_{i}$, which are denoted by the blue circles in Fig. 1(a), whose radii are $\mathcal{R}_{T_{i}}$ for $i \in\{1,2, \ldots, N\}$. This assumption can allow the LEDs to maximize the received optical power at UEs' sites given a specific number of LEDs.

\section{B. Received Optical Power Density Analysis}

According to [9], the optical channel gain between an LED transmitter and a receiver in VLC systems can be written as

$$
G=\left\{\begin{array}{cl}
\frac{(m+1)}{2 \pi l^{2}} A_{\mathrm{RX}} \cos ^{m}(\phi) \cos (\psi) & \text { for }|\psi| \leq \Psi, \\
0 & \text { for }|\psi|>\Psi
\end{array}\right.
$$

where $m=-\ln (2) / \ln \left(\cos \left(\phi_{1 / 2}\right)\right)$ is the order of Lambertian emission with half illuminance at $\phi_{1 / 2}$. The parameter $l$ signifies the distance between the LED and the receiver, and $\phi$ and $\psi$ denote the angle of irradiance and the angle of incidence between the transmitter and the receiver. Also, the receiver collection area is given by $A_{\mathrm{RX}}=\kappa^{2} A_{\mathrm{PD}} / \sin ^{2}(\Psi)\left[\mathrm{m}^{2}\right]$, where $\kappa$ is the refractive index of the optical concentrator, $A_{\mathrm{PD}}\left[\mathrm{m}^{2}\right]$ is the physical area of the PD, and $\Psi$ is the received field of view of the PD. Moreover, as in [3], [4], if we assume that a receiver's PD faces up normal to the work plane, we can rewrite (1) as

$$
G=\frac{(m+1)}{2 \pi l^{2}} A_{\mathrm{RX}}\left(\frac{H}{l}\right)^{m}\left(\frac{H}{l}\right)=\varrho l^{-(m+3)}
$$


where $\varrho=(m+1) A_{\mathrm{RX}} H^{(m+1)} / 2 \pi$. Note that (2) is valid only when $|\psi| \leq \Psi$ is satisfied.

In the continuum model, to deal with an infinite number of LEDs, we characterize the emitted optical power of LEDs by using the average optical power density per unit of LED area $P_{T}\left[\mathrm{~W} / \mathrm{m}^{2}\right]$. We assume that the LED transmitters emit signals with the same average optical power density $P_{T}$. Utilizing the channel gain model in (2), the average received optical power density of the signal emitted by the LED transmitters for $\mathrm{UE}_{i}$ $P_{i}\left[\mathrm{~W} / \mathrm{m}^{2}\right]$ at a point $(r, \theta)$ in the work plane, the origin of which is $\mathrm{UE}_{i}$, can be described by

$$
\begin{gathered}
P_{i}(r, \theta)=\int_{0}^{\mathcal{R}_{T_{i}}} \int_{0}^{2 \pi} P_{T} \frac{(m+1)}{2 \pi l_{T_{i}}^{2}}\left(\frac{H}{l_{T_{i}}}\right)^{(m+1)} r_{T_{i}} \mathrm{~d} \theta_{T_{i}} \mathrm{~d} r_{T_{i}} \\
\stackrel{(a)}{=} \frac{P_{T}}{2}\left(1+\frac{\mathcal{R}_{T_{i}}^{2}-H^{2}-r^{2}}{\sqrt{\left(H^{2}+r^{2}\right)^{2}+2 \mathcal{R}_{T_{i}}^{2}\left(H^{2}-r^{2}\right)+\mathcal{R}_{T_{i}}^{4}}}\right)
\end{gathered}
$$

where $l_{T_{i}}=\sqrt{r_{T_{i}}^{2}+r^{2}-2 r_{T_{i}} r \cos \left(\theta_{T_{i}}-\theta\right)+H^{2}}$ denotes the distance between the differential information transmitter and the point $(r, \theta)$ in the work plane. For (a) and the following analysis, we assume all the LED transmitters have the Lambertian emission pattern with $\phi_{1 / 2}=60^{\circ}(m=1)$. Since the value of $P_{i}(r, \theta)$ is independent of $\theta$, we replace the notation of $P_{i}(r, \theta)$ with $P_{i}(r)$. Also, note that $P_{i}(0)$ and $P_{i}\left(r_{E_{i}}\right)$ denote the average received optical power density of the data signal at the $\mathrm{UE}_{i}$ and $\mathrm{ED}_{i}$ sites, respectively.

\section{Data Transmission}

The data current signal $x_{i}(t) \in[-1,1]$ for $i \in\{1,2, \ldots, N\}$ to be transmitted to $\mathrm{UE}_{i}$ in time slot $t$ is generated from a certain real constellation, e.g., a DC-biased pulse amplitude modulation (PAM) scheme, and multiplied by a modulation index $\alpha \in[0,1]$ and a fixed bias current $I_{\mathrm{DC}} \in \mathbb{R}^{+}$, where $\mathbb{R}^{+}$ denotes the set of non-negative real-valued numbers. Thus, the modulated signal $s_{i}(t)$ can be described as $s_{i}(t)=\alpha I_{\mathrm{DC}} x_{i}(t)$. To maintain linear current-to-light conversion and avoid clipping distortion, the LED transmitter has an amplitude constraint on its input power, i.e., $s_{i}(t)$ is subject to the amplitude constraint $\left|s_{i}(t)\right| \leq \alpha I_{\mathrm{DC}}$. Therefore, the emitted optical power of each LED can be $P_{\mathrm{TX}, i}(t)=\eta\left(I_{\mathrm{DC}}+s_{i}(t)\right)$ [W], where $\eta$ $[W / A]$ is the current-to-light conversion efficiency. $P_{\mathrm{TX}, i}(t)$ is also related to the average optical power density $P_{T}$ of the continuum model as $P_{\mathrm{TX}, i}(t)=P_{T} \hat{x}_{i}(t) / \lambda_{T}$, where $\lambda_{T}$ is the density of LED transmitters and $\hat{x}_{i}(t)=x_{i}(t) /(1[A])$ is the normalized (dimensionless) version of $x_{i}(t)$. Also, $\mathbb{E}\left[s_{i}(t)\right]=0$ is assumed, the modulated signal does not affect illumination.

Using the optical power density expression (3), the received signal voltages $y_{U_{i}}(t)$ and $y_{E_{i}}(t)$ for $i \in\{1,2, \ldots, N\}$ at $\mathrm{UE}_{i}$ and $\mathrm{ED}_{i}$ after removing the DC bias can be described as

$$
y_{U_{i}}(t)=\zeta_{U_{i}} P_{i}(0) \hat{x}_{i}(t)+\sum_{j=1, j \neq i}^{N} \zeta_{U_{i}} P_{j}\left(r_{U_{i}, U_{j}}\right) \hat{x}_{j}(t)+n_{U_{i}}(t),
$$

$$
y_{E_{i}}(t)=\zeta_{E_{i}} P_{i}\left(r_{E_{i}}\right) \hat{x}_{i}(t)+\sum_{j=1, j \neq i}^{N} \zeta_{E_{i}} P_{j}\left(r_{E_{i}, U_{j}}\right) \hat{x}_{j}(t)+n_{E_{i}}(t)
$$

respectively, where $\zeta_{k}=\alpha A_{\mathrm{PD}, k} \kappa_{k}^{2} R_{\mathrm{rsp}, k} T_{k} / \sin ^{2}\left(\Psi_{k}\right)\left[\mathrm{m}^{2} \cdot \mathrm{V} / \mathrm{W}\right]$ for $k \in\left\{U_{i}, E_{i}\right\} . R_{\mathrm{rsp}, k}[\mathrm{~A} / \mathrm{W}]$ is the photodetector's responsivity and $T_{k}$ [V/A] is the transimpedance amplifier gain. Also, $r_{U_{i}, U_{j}}$ and $r_{E_{i}, U_{j}}$ denote the distances between $\mathrm{UE}_{i}$ and $\mathrm{UE}_{j}$ and between $\mathrm{ED}_{i}$ and $\mathrm{UE}_{j}$, respectively, where

$r_{E_{i}, U_{j}}=\sqrt{r_{U_{i}, U_{j}}^{2}+r_{E_{i}}^{2}-2 r_{U_{i}, U_{j}} r_{E_{i}} \cos \left(\theta_{E_{i}}-\theta_{U_{i}, U_{j}}\right)}$. As seen in (4), the first terms in both equations are the desired signals for $\mathrm{UE}_{i}$ and $\mathrm{ED}_{i}$, respectively, while the second term is the multiuser interference (MUI). Also, $n_{k}(t)$ signifies zero-mean additive white Gaussian noise (AWGN) with variance $\sigma^{2}$. For notational convenience, the time index $t$ is ignored for the remainder of the paper.

\section{Performance Measures}

For a multiuser system, the secrecy capacity (or rate) region is a useful metric for measuring the secrecy performance of multiple users [11]. However, due to its high mathematical complexity, in this paper, we utilize a practical approach of considering the secrecy capacity of the single secured user by treating the other ordinary users as interference. This approach is consistent with the assumption in Section II-A that the number of users that require the secured PLS transmission can be limited to one. Thus, the secrecy capacity for $\mathrm{UE}_{i}$ in the multiuser VLC systems is given by [2]

$$
C_{S_{i}}=\max _{p_{x_{i}}}\left(\mathbb{I}\left(x_{i} ; y_{U_{i}}\right)-\mathbb{I}\left(x_{i} ; y_{E_{i}}\right)\right), \quad \text { s.t. } \quad\left|x_{i}\right| \leq 1
$$

where $\mathbb{I}(\cdot ; \cdot)$ denotes the mutual information and $p_{x_{i}}$ is the input distribution of $x_{i}$. It is not feasible to deduce a closed-form solution for (5) due to the amplitude constraint [12]. It was shown in [12], [13] that the optimal probability distribution $p_{x_{i}}^{*}$, i.e., the solution of the optimization problem (5), is unique and discrete with a finite support set, thus it can be efficiently computed using numerical methods. Nevertheless, closed-form expressions are often preferred for engineering systems. Thus, in the following, we provide an achievable secrecy rate expression for the multiuser VLC system.

To simplify the derivation of a closed-form achievable secrecy rate expression, we assume that the data signal $x_{i}$ (and $\hat{x}_{i}$ ) follows the truncated Gaussian distribution $\mathcal{N}_{T}\left(0, \sigma_{T}^{2}\right)$ defined over $[-1,1]$, where $\sigma_{T} \in \mathbb{R}_{+}$, as in [5]. Its probability density function (PDF) is given by

$$
f\left(x_{i}\right)=\phi\left(\frac{x_{i}}{\sigma_{T}}\right) /\left(\Phi\left(\frac{1}{\sigma_{T}}\right)-\Phi\left(\frac{-1}{\sigma_{T}}\right)\right)
$$

where $\phi(v)=e^{-v^{2} / 2} / \sqrt{2 \pi}$ and $\Phi(\omega)=(1+\operatorname{erf}(\omega / \sqrt{2})) / 2$. The error function $\operatorname{erf}(\cdot)$ is defined as $\operatorname{erf}(\omega)=1 / \sqrt{\pi} \int_{-\omega}^{\omega} e^{-t^{2}} \mathrm{~d} t$. Note that truncated Gaussian distribution was shown to outperform the uniform distribution in terms of secrecy rates in VLC systems [5]. With these assumptions, we present the following lemma, which provides an analytic achievable secrecy rate expression for the system in question. 
Lemma 1. An achievable secrecy rate for $U E_{i}$ in the Gaussian wiretap multiuser channel of (4) is given by

$$
\begin{aligned}
R_{S_{i}}= & \max \left\{\frac{1}{2} \log \left(\frac{e^{2 \eta}\left(P_{i}^{2}(0)+\sum_{j=1, j \neq i}^{N} P_{j}^{2}\left(r_{U_{i}, U_{j}}\right)\right)+C}{\varphi \sum_{j=1, j \neq i}^{N} P_{j}^{2}\left(r_{U_{i}, U_{j}}\right)+C}\right)\right. \\
& \left.-\frac{1}{2} \log \left(\frac{\varphi\left(P_{i}^{2}\left(r_{E_{i}}\right)+\sum_{j=1, j \neq i}^{N} P_{j}^{2}\left(r_{E_{i}, U_{j}}\right)\right)}{e^{2 \eta} \sum_{j=1, j \neq i}^{N} P_{j}^{2}\left(r_{E_{i}, U_{j}}\right)}\right), 0\right\}
\end{aligned}
$$

where

$$
\begin{aligned}
& \eta=\log (Z)+\left(-\frac{1}{\sigma_{T}} \phi\left(\frac{-1}{\sigma_{T}}\right)-\frac{1}{\sigma_{T}} \phi\left(\frac{1}{\sigma_{T}}\right)\right) / 2 Z, \\
& \varphi=1+\frac{\frac{-1}{\sigma_{T}} \phi\left(\frac{-1}{\sigma_{T}}\right)-\frac{1}{\sigma_{T}} \phi\left(\frac{1}{\sigma_{T}}\right)}{Z}-\left(\frac{\phi\left(\frac{-1}{\sigma_{T}}\right)-\phi\left(\frac{1}{\sigma_{T}}\right)}{Z}\right)^{2}, \\
& Z=\Phi\left(\frac{1}{\sigma_{T}}\right)-\Phi\left(\frac{-1}{\sigma_{T}}\right), \quad C=\sigma^{2} / \zeta_{U_{i}}^{2} \sigma_{T}^{2} .
\end{aligned}
$$

Proof. See Appendix A.

A corollary is that an achievable rate for $\mathrm{UE}_{i}$ under the assumption of the truncated Gaussian distribution on the input signal $x_{i}$ is given by

$$
R_{i}=\frac{1}{2} \log \left(\frac{e^{2 \eta}\left(P_{i}^{2}(0)+\sum_{j=1, j \neq i}^{N} P_{j}^{2}\left(r_{U_{i}, U_{j}}\right)\right)+C}{\varphi \sum_{j=1, j \neq i}^{N} P_{j}^{2}\left(r_{U_{i}, U_{j}}\right)+C}\right) .
$$

\section{Optimal LED Selection for Multiuser VLC SYSTEMS}

In this section, we investigate an optimization problem based on the secrecy rate objective. In the considered system model, there are a target UE, who should be protected by using the PLS technique, its corresponding ED, and multiple ordinary UEs. Therefore, it can be a natural objective to maximize the secrecy rate of the target user, subject to a constraint on the required data rates for the ordinary users. Moreover, since we do not know the precise location of the $\mathrm{ED}$, we consider the average secrecy rate with respect to its position, i.e., $\mathbb{E}\left[R_{S_{i}}\right]$.

Firstly, the average secrecy rate $\mathbb{E}\left[R_{S_{i}}\right]$ can be numerically calculated as (9) at the top of the next page, where $r_{E_{i}, U_{j}}(r, \theta)=\sqrt{r_{U_{i}, U_{j}}^{2}+r^{2}-2 r_{U_{i}, U_{j}} r \cos \left(\theta-\theta_{U_{i}, U_{j}}\right)}$ and $f_{r_{E_{i}}, \theta_{E_{i}}}(r, \theta)$ is the joint PDF of $\left(r_{E_{i}}, \theta_{E_{i}}\right)$. For example, $f_{r_{E_{i}}, \theta_{E_{j}}}(r, \theta)$ for the uniform distribution and the bivariate normal distribution of the ED location can be written as

$$
\begin{aligned}
& f_{r_{E_{i}}, \theta_{E_{i}}}^{\text {unif. }}(r, \theta)=1 / \pi \mathcal{R}_{E_{i}}^{2} \text { for }\left\{\begin{array}{l}
0 \leq r<\mathcal{R}_{E_{i}} \\
0 \leq \theta<2 \pi
\end{array}\right. \\
& f_{r_{E_{i}}, \theta_{E_{i}}}^{\text {norm. }}(r, \theta)=\exp \left(\frac{-r^{2}}{2 \sigma_{E_{i}}^{2}}\right) /\left(2 \pi \sigma_{E_{i}}^{2}\right) \quad \text { for }\left\{\begin{array}{l}
0 \leq r<\infty \\
0 \leq \theta<2 \pi
\end{array}\right.
\end{aligned}
$$

respectively, where $\sigma_{E_{i}}^{2}$ in (10b) is the variance of the $\mathrm{ED}$ location in a Cartesian coordinate system, i.e., $x=r \cos \theta$ and $y=r \sin \theta$ with $X \sim \mathcal{N}\left(0, \sigma_{E_{i}}^{2}\right), Y \sim \mathcal{N}\left(0, \sigma_{E_{i}}^{2}\right)$ and their correlation is assumed to be as $\operatorname{cor}(X, Y)=0$.

Utilizing the average secrecy rate (9), we formulate the optimization problem as

$$
\begin{gathered}
\bar{R}_{s_{i}}^{\star}=\max _{\mathcal{R}_{T_{j}} \text { for }} \bar{R}_{j \in\{1,2, \ldots, N\}} \\
\text { s.t. }\left\{\begin{array}{l}
R_{j} \geq \rho_{j} \text { for } \forall j \in\{1,2, \ldots, N\}, j \neq i \\
\mathcal{R}_{T_{j}}+\mathcal{R}_{T_{k}} \leq r_{U_{j}, U_{k}} \text { for } \forall j, k \in\{1,2, \ldots, N\}, j \neq k \\
\mathcal{R}_{T_{j}} \geq 0 \text { for } \forall j \in\{1,2, \ldots, N\} .
\end{array}\right.
\end{gathered}
$$

Note that since we utilize the continuous LED model, finding the optimal set of the LED transmitters is transformed to finding the optimal radii of the LED circles. Also, note that the average optical power density $P_{i}(\cdot)$ for $i \in\{1,2, \ldots, N\}$ in (9) is a function of $\mathcal{R}_{T_{i}}$ as shown in (3). Here, $\left\{\rho_{j}\right\}$ signify the target constraints on the required data rates for the ordinary users. Also, the second constraint ensures that the LED transmitter circles do not overlap. This optimization problem is a non-convex problem and the objective function includes the integral to be solved numerically. However, the number of the optimization variables is $N$ and the objective function includes only a two-dimensional integral, where all the integrating functions are closed-form. Thus, using a standard optimization programming like the sequential quadratic programming (SQP) algorithm [14], we can efficiently solve the problem with a proper initial point, which can be effectively anticipated by considering the relative locations of the UEs and the statistical information on the ED location. For example, when two UEs are located near to each other, the optimal radii of LEDs for the two UEs should be small not to cause severe interference to another UE. When $N=3$, for example, solving the problem requires from 10 to 30 seconds on a standard PC (Intel i7, $3.4 \mathrm{GHz}$ ) using MATLAB.

\section{Numerical Results}

In this section, numerical results are given to verify the performance of the proposed LED selection scheme in multiuser VLC systems. We assume that three UEs are present, where $\mathrm{UE}_{1}$ is the target user; thus $\mathrm{ED}_{1}$ is present trying to eavesdrop $\mathrm{UE}_{1}$. The locations of $\mathrm{UE}_{i}$ for $i \in\{1,2,3\}$ in the work plane are $(0,0),\left(0, y_{\mathrm{UE}_{2}}\right),\left(x_{\mathrm{UE}_{3}}, 0\right)$, respectively. Also, we set $H=2.2 \mathrm{~m}, P_{T}=1 \mathrm{~W} / \mathrm{m}^{2}, \alpha=0.5, \kappa=1.5, A_{\mathrm{PD}}=1 \mathrm{~cm}^{2}$, $\Psi=90^{\circ}, \sigma^{2}=1.47 \times 10^{-13} A^{2}$, and $\sigma_{T}=0.62$.

To see how the interference caused by multiple users affects the PLS transmission, Fig. 2 shows the optimized average secrecy rate of $\mathrm{UE}_{1} \bar{R}_{S_{1}}^{\star}$ for the different locations of ordinary UEs. Here, we assume that $\mathrm{ED}_{1}$ is uniformly (randomly) located within a circle with radius $R_{E_{1}}=5 \mathrm{~m}$ centered at $\mathrm{UE}_{1}$. In the figure, it is noted that $\bar{R}_{S_{1}}^{\star}$ can be higher as the ordinary users $\mathrm{UE}_{2}$ and $\mathrm{UE}_{3}$ are located farther away from $\mathrm{UE}_{1}$. More specifically, when $\mathrm{UE}_{3}$ is near to $\mathrm{UE}_{1}$ (less than $2.7 \mathrm{~m}$ ), $\bar{R}_{S_{1}}^{\star}$ is almost zero regardless of the location of $\mathrm{UE}_{2}$ due to the strong interference caused by the signal for $\mathrm{UE}_{3}$. In contrast, when $\mathrm{UE}_{2}$ is far from $\mathrm{UE}_{1}$, e.g., when $y_{U E_{2}}=6 \mathrm{~m}, \bar{R}_{S_{1}}^{\star}$ increases as $\mathrm{UE}_{3}$ moves away from $\mathrm{UE}_{1}$, i.e., as $x_{U E_{3}}$ increases. This result indicates that the transmissions from the LEDs to $\mathrm{UE}_{2}$ and 


$$
\begin{aligned}
& \bar{R}_{S_{i}}=\mathbb{E}\left[R_{S_{i}}\right]=\int_{0}^{\mathcal{R}_{E_{i}}} \int_{0}^{2 \pi} f_{r_{E_{i}}, \theta_{E_{i}}}(r, \theta) R_{S_{i}}(r, \theta) r \mathrm{~d} \theta \mathrm{d} r \\
& =\int_{0}^{\mathcal{R}_{E_{i}}} \int_{0}^{2 \pi} f_{r_{E_{i}}, \theta_{E_{i}}}(r, \theta) \max \left\{\frac{1}{2} \log \left(\frac{e^{2 \eta}\left(P_{i}^{2}(0)+\sum_{j=1, j \neq i}^{N} P_{j}^{2}\left(r_{U_{i}, U_{j}}\right)\right)+C}{\varphi \sum_{j=1, j \neq i}^{N} P_{j}^{2}\left(r_{U_{i}, U_{j}}\right)+C}\right)-\frac{1}{2} \log \left(\frac{\varphi\left(P_{i}^{2}(r)+\sum_{j=1, j \neq i}^{N} P_{j}^{2}\left(r_{E_{i}, U_{j}}(r, \theta)\right)\right)}{e^{2 \eta} \sum_{j=1, j \neq i}^{N} P_{j}^{2}\left(r_{E_{i}, U_{j}}(r, \theta)\right)}\right), 0\right\} r \mathrm{~d} \theta \mathrm{d} r
\end{aligned}
$$

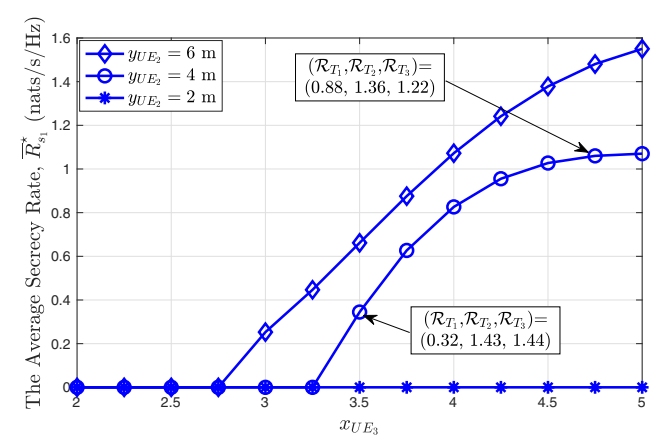

Fig. 2. The optimized average secrecy rate of $U_{1}$ for the different locations of ordinary UEs. $\mathrm{ED}_{1}$ is assumed to be present trying to eavesdrop $\mathrm{UE}_{1}$. Three UEs are present, where the locations of $\mathrm{UE}_{i}$ for $i \in\{1,2,3\}$ in the work plane are $(0,0),\left(0, y_{\mathrm{UE}_{2}}\right),\left(x_{\mathrm{UE}_{3}}, 0\right)$, respectively. The uniform distribution of the ED location in (10a) with $R_{E_{1}}=5 \mathrm{~m}$ is adopted. $\rho_{j}=3$ nats $/ \mathrm{s} / \mathrm{Hz}$ for $j \in\{2,3\}$ is used.

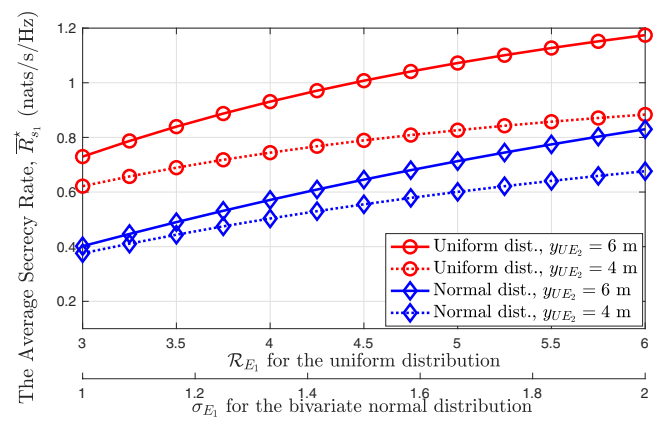

Fig. 3. The optimized average secrecy rate of $\mathrm{UE}_{1}$ for the different distributions of $\mathrm{ED}_{1}$. Three UEs are present, where the locations of $\mathrm{UE}_{i}$ for $i \in\{1,2,3\}$ in the work plane are $(0,0),\left(0, y_{\mathrm{UE}_{2}}\right),(4,0)$, respectively. $\rho_{j}=3$ nats $/ \mathrm{s} / \mathrm{Hz}$ for $j \in\{2,3\}$ is used.

$\mathrm{UE}_{3}$ cause varying degrees of interference at $\mathrm{UE}_{1}$ according to the distances between the target and ordinary users, which significantly affects the average secrecy rate. In addition, the optimal radii of LED circles $\mathcal{R}_{T_{i}}$ for $i \in\{1,2,3\}$ are provided for two cases to show how the proposed scheme effectively manipulates the MUI and the information signal reception. When $y_{\mathrm{UE}_{2}}=4 \mathrm{~m}$ and $x_{\mathrm{UE}_{3}}=3.5 \mathrm{~m}$, since the ordinary users are near to $\mathrm{UE}_{1}, \mathcal{R}_{T_{1}}$ is relatively small $(0.32)$ not to cause severe interference at $\mathrm{UE}_{2}$ and $\mathrm{UE}_{3}$ sites. In contrast, as $\mathrm{UE}_{3}$ moves away from $\mathrm{UE}_{1}, \mathcal{R}_{T_{1}}$ largely increases to 0.88 to increase the signal reception at $\mathrm{UE}_{1}$, accordingly, and improve the average secrecy rate.

Fig. 3 shows $\bar{R}_{s_{1}}^{\star}$ for the different distributions of $\mathrm{ED}_{1}$. The uniform distribution and the bivariate normal distribution in (10a) and (10b), respectively, are used. When $\mathcal{R}_{E_{1}}$ and $\sigma_{E_{1}}$ are small, in other words, $\mathrm{ED}_{1}$ is likely to be nearer to $\mathrm{UE}_{1}, \mathrm{ED}_{1}$

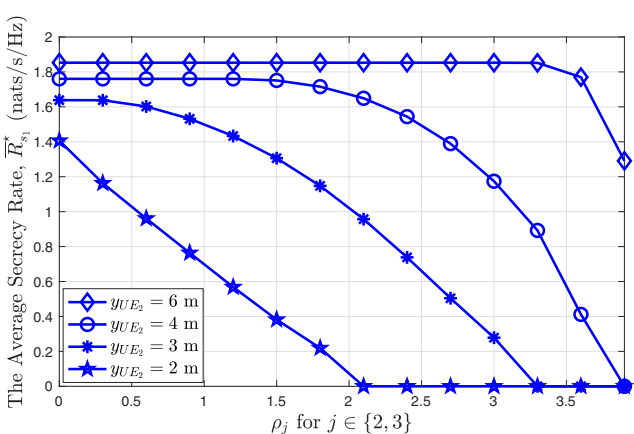

Fig. 4. The optimized average secrecy rate of $\mathrm{UE}_{1}$ for the target constraints on the required data rates for the ordinary users $\rho_{j}$ for $j \in\{2,3\}$. Three UEs are present, where the locations of $\mathrm{UE}_{i}$ for $i \in\{1,2,3\}$ in the work plane are $(0,0),\left(0, y_{\mathrm{UE}_{2}}\right),(6,0)$, respectively.

can easily overhear the information signal; thus $\bar{R}_{S_{1}}^{\star}$ is shown to be low. In contrast, as $\mathcal{R}_{E_{1}}$ and $\sigma_{E_{1}}$ increase, $\bar{R}_{s_{1}}^{\star}$ increases as well. Also, for example, if $\mathrm{ED}_{1}$ is randomly located with the uniform distribution with $\mathcal{R}_{E_{1}}=3 \mathrm{~m}, \mathrm{ED}_{1}$ is present in a circle with radius $3 \mathrm{~m}$ centered at $\mathrm{UE}_{1}$ with a $100 \%$ probability. Meanwhile, $\mathrm{ED}_{1}$ with the bivariate normal distribution with $\sigma_{E_{1}}=1 \mathrm{~m}$ is present in a circle with radius $3 \mathrm{~m}$ with a 98\% probability. Comparing $\bar{R}_{S_{1}}^{\star}$ when the uniform distribution with $\mathcal{R}_{E_{1}}=3 \mathrm{~m}$ and the bivariate normal distribution with $\sigma_{E_{1}}=1 \mathrm{~m}$ are adopted, $\bar{R}_{S_{1}}^{\star}$ for the uniform distribution is almost twice the rate attainable under the bivariate normal distribution assumption. Thus, from this result, we can note that even when the ED is located within the same circular boundary, the spatial distribution of the ED largely affects the average secrecy rate.

Fig. 4 shows $\bar{R}_{s_{1}}^{\star}$ for the different required data rates $\rho_{j}$ for $j \in\{2,3\}$. It is shown that when the ordinary user $\mathrm{UE}_{2}$ is near $\mathrm{UE}_{1}$, e.g., when $y_{U E_{2}}=2 \mathrm{~m}, \bar{R}_{S_{1}}^{\star}$ decreases as $\rho_{j}$ increases. This is because higher rate constraints require more LEDs to transmit information signals, which in turn causes a significant amount of interference at $\mathrm{UE}_{1}$. On the other hand, when the ordinary users $\mathrm{UE}_{2}$ and $\mathrm{UE}_{3}$ are far away from $\mathrm{UE}_{1}$, e.g., when they are located at $(0,6)$ and $(6,0)$, respectively, the average secrecy rate does not decrease even as $\rho_{j}$ increases up to 3.2 nats $/ \mathrm{s} / \mathrm{Hz}$. This result indicates that the relative locations of the multiple users and the required data rates are the essential factors that affect the average secrecy rate.

\section{CONCLUSION}

In this paper, we proposed a novel LED selection scheme for multiuser VLC systems. By utilizing the continuous LED 
model, a related optimization problem was formulated to find the optimal set of LEDs that maximizes the average secrecy rate for the target user, while satisfying the required data rates for ordinary users. Numerical simulations verified that, in multiuser VLC systems, the interference caused by other users' signals should be carefully considered to improve secrecy performance, which is significantly affected by the relative locations of multiple UEs, the spatial distribution of a random $\mathrm{ED}$, and the required data rates for ordinary users. Based on the results, we can expect that MISO beamforming strategies, which can significantly mitigate interference by using welldesigned precoding matrix, can be future research topics to improve the secrecy performance in multiuser VLC systems.

\section{APPENDIX A}

\section{DERIVATION OF THE SECRECY RATE}

A lower bound on the secrecy rate of (5) can be obtained as follows

$$
\begin{aligned}
C_{S_{i}} & \left.=\max _{p_{x_{i}}}\left(\mathbb{I}\left(x_{i} ; y_{U_{i}}\right)-\mathbb{I}\left(x_{i} ; y_{E_{i}}\right)\right)=\max _{p_{\hat{x}_{i}}} \mathbb{I}\left(\hat{x}_{i} ; y_{U_{i}}\right)-\mathbb{I}\left(\hat{x}_{i} ; y_{E_{i}}\right)\right) \\
& \stackrel{(a)}{\geq} \mathbb{I}\left(\hat{x}_{i} ; y_{U_{i}}\right)-\mathbb{I}\left(\hat{x}_{i} ; y_{E_{i}}\right) \stackrel{(b)}{\geq} \mathbb{I}\left(\hat{x}_{i} ; y_{U_{i}}\right)-\mathbb{I}\left(\hat{x}_{i} ; v_{E_{i}}\right) \\
& =\mathbb{h}\left(y_{U_{i}}\right)-\mathbb{h}\left(y_{U_{i}} \mid \hat{x}_{i}\right)-\mathbb{h}\left(v_{E_{i}}\right)+\mathbb{h}\left(v_{E_{i}} \mid \hat{x}_{i}\right)
\end{aligned}
$$

where $\mathrm{h}(\cdot)$ denotes differential entropy and $v_{E_{i}}=$ $\zeta_{E_{i}} P_{i}\left(r_{E_{i}}\right) \hat{x}_{i}+\sum_{j=1, j \neq i}^{N} \zeta_{E_{i}} P_{j}\left(r_{E_{i}, U_{j}}\right) \hat{x}_{j}$. (a) follows from dropping the maximization by choosing a truncated Gaussian distribution on $p_{\hat{x}_{i}}$, and (b) follows from the data-processing inequality, i.e., $y_{E_{i}}=g\left(v_{E_{i}}\right)=v_{E_{i}}+N_{E_{i}}$. Firstly, we lowerbound $\mathrm{h}\left(y_{U_{i}}\right)$ by using the entropy-power inequality as

$$
\begin{aligned}
& \operatorname{hh}\left(y_{U_{i}}\right) \geq \\
& \frac{1}{2} \log \left(e^{2 \mathrm{~h}\left(\zeta U_{i} P_{i}(0) \hat{x}_{i}\right)}+\sum_{j=1, j \neq i}^{N} e^{2 \mathrm{~h}\left(\zeta_{U_{i}} P_{j}\left(r_{U_{i}, U_{j}}\right) \hat{x}_{j}\right)}+e^{2 \mathrm{~h}\left(N_{U_{i}}\right)}\right) \\
& =\frac{1}{2} \log \left(2 \pi e\left(\sigma_{T}^{2} \zeta_{U_{i}}^{2} e^{2 \eta}\left(P_{i}^{2}(0)+\sum_{j=1, j \neq i}^{N} P_{j}^{2}\left(r_{U_{i}, U_{j}}\right)\right)+\sigma^{2}\right)\right)
\end{aligned}
$$

where (17) follows from the facts that

$$
\begin{aligned}
& \text { h }\left(\zeta_{U_{i}} P_{i}(0) \hat{x}_{i}\right)=\log \left(\zeta_{U_{i}} P_{i}(0)\right)+\frac{1}{2} \log \left(2 \pi e \sigma_{T}^{2}\right)+\eta, \\
& \text { h }\left(\zeta_{U_{i}} P_{j}\left(r_{U_{i}, U_{j}}\right) \hat{x}_{j}\right)=\log \left(\zeta_{U_{i}} P_{j}\left(r_{U_{i}, U_{j}}\right)\right)+\frac{1}{2} \log \left(2 \pi e \sigma_{T}^{2}\right)+\eta, \\
& \mathbb{h}\left(N_{U_{i}}\right)=\frac{1}{2} \log 2 \pi e \sigma^{2} .
\end{aligned}
$$

Then, we upper-bound $\mathbb{h}\left(y_{U_{i}} \mid \hat{x}_{i}\right)$ and $\mathbb{h}\left(v_{E_{i}}\right)$ as

$$
\begin{aligned}
& \mathbb{h}\left(y_{U_{i}} \mid \hat{x}_{i}\right)=\mathbb{h}\left(y_{U_{i}}-\zeta_{U_{i}} P_{i}(0) \hat{x}_{i} \mid \hat{x}_{i}\right) \\
= & \mathbb{h}\left(\sum_{j=1, j \neq i}^{N} \zeta_{U_{i}} P_{j}\left(r_{U_{i}, U_{j}}\right) \hat{x}_{j}+N_{U_{i}}\right) \\
\leq & \frac{1}{2} \log 2 \pi e\left(\sigma_{T}^{2} \zeta_{U_{i}}^{2} \varphi \sum_{j=1, j \neq i}^{N} P_{j}^{2}\left(r_{U_{i}, U_{j}}\right)+\sigma^{2}\right), \\
\mathbb{h}\left(v_{E_{i}}\right) & =\mathbb{h}\left(\zeta_{E_{i}} P_{i}\left(r_{E_{i}}\right) \hat{x}_{i}+\sum_{j=1, j \neq i}^{N} \zeta_{E_{i}} P_{j}\left(r_{E_{i}, U_{j}}\right) \hat{x}_{j}\right) \\
\leq & \frac{1}{2} \log \left(2 \pi e \sigma_{T}^{2} \zeta_{E_{i}}^{2} \varphi\left(P_{i}^{2}\left(r_{E_{i}}\right)+\sum_{j=1, j \neq i}^{N} P_{j}^{2}\left(r_{E_{i}, U_{j}}\right)\right)\right)
\end{aligned}
$$

by using the differential entropy of Gaussian random variables with variances $\operatorname{var}\left\{\sum_{j=1, j \neq i}^{N} \zeta_{U_{i}} P_{j}\left(r_{U_{i}, U_{j}}\right) \hat{x}_{j}+N_{U_{i}}\right\}$ and $\operatorname{var}\left\{\zeta_{E_{i}} P_{i}\left(r_{E_{i}}\right) \hat{x}_{i}+\sum_{j=1, j \neq i}^{N} \zeta_{E_{i}} P_{j}\left(r_{E_{i}, U_{j}}\right) \hat{x}_{j}\right\}, \quad$ respectively. Lastly, we lower-bound $\mathbb{h}\left(v_{E_{i}} \mid \hat{x}_{i}\right)$, once again, by using the entropy-power inequality as

$$
\begin{aligned}
\mathbb{h}\left(v_{E_{i}} \mid \hat{x}_{i}\right) & =\mathbb{h}\left(v_{E_{i}}-\zeta_{E_{i}} P_{i}\left(r_{E_{i}}\right) \hat{x}_{i} \mid \hat{x}_{i}\right)=\mathbb{h}\left(\sum_{j=1, j \neq i}^{N} \zeta_{E_{i}} P_{j}\left(r_{E_{i}, U_{j}}\right) \hat{x}_{j}\right) \\
& \geq \frac{1}{2} \log \left(\sum_{j=1, j \neq i}^{N} e^{2 \mathbb{h}\left(\zeta_{E_{i}} P_{j}\left(r_{E_{i}, U_{j}}\right) \hat{x}_{j}\right)}\right) \\
& =\frac{1}{2} \log \left(2 \pi e \sigma_{T}^{2} \zeta_{E_{i}}^{2} e^{2 \eta} \sum_{j=1, j \neq i}^{N} P_{j}^{2}\left(r_{E_{i}, U_{j}}\right)\right)
\end{aligned}
$$

Plugging (13), (15), (16) and (17) into (12) yields the secrecy rate for the multiuser VLC system in (7).

\section{ACKNOWLEDGMENT}

This work was supported by EPSRC grant number EP/R006377/1(“M3NETs").

\section{REFERENCES}

[1] D. Karunatilaka, F. Zafar, V. Kalavally, and R. Parthiban, "LED based indoor visible light communications: State of the art," IEEE Commun. Surveys Tuts., vol. 17, no. 3, pp. 1649-1678, 2015.

[2] M. Bloch and J. Barros, Physical-Layer Security: From Information Theory to Security Engineering. Cambridge University Press, 2011.

[3] A. Mostafa and L. Lampe, "Physical-layer security for MISO visible light communication channels," IEEE J. Sel. Areas Commun., vol. 33, no. 9, pp. 1806-1818, Sep. 2015.

[4] S. Cho, G. Chen, and J. P. Coon, "Securing visible light communication systems by beamforming in the presence of randomly distributed eavesdroppers," IEEE Trans. Wireless Commun., vol. 17, no. 5, pp. 29182931, May 2018.

[5] H. Zaid, Z. Rezki, A. Chaaban, and M. S. Alouini, "Improved achievable secrecy rate of visible light communication with cooperative jamming," in IEEE GlobalSIP in Orlando, U.S.A., Dec. 2015, pp. 1165-1169.

[6] T. V. Pham and A. T. Pham, "On the secrecy sum-rate of MU-VLC broadcast systems with confidential messages," in 2016 CSNDSP in Prague, Czech Republic, Jul. 2016, pp. 1-6.

[7] M. A. Arfaoui, A. Ghrayeb, and C. M. Assi, "Secrecy performance of multi-user MISO VLC broadcast channels with confidential messages," IEEE Trans. Wireless Commun., vol. 17, no. 11, pp. 7789-7800, Nov. 2018.

[8] X. Zhao, H. Chen, and J. Sun, "On physical-layer security in multiuser visible light communication systems with non-orthogonal multiple access," IEEE Access, vol. 6, pp. 34004-34 017, 2018.

[9] T. Komine and M. Nakagawa, "Fundamental analysis for visible-light communication system using LED lights," IEEE Trans. Consum. Electron., vol. 50, no. 1, pp. 100-107, Feb. 2004.

[10] S. Cho, G. Chen, and J. P. Coon, "Enhancement of physical layer security with simultaneous beamforming and jamming for visible light communication systems," IEEE Trans. Inf. Forens. Security, vol. 14, no. 10, pp. 2633-2648, Oct. 2019.

[11] R. Liu, I. Maric, P. Spasojevic, and R. D. Yates, "Discrete memoryless interference and broadcast channels with confidential messages: Secrecy rate regions," IEEE Trans. Inf. Theory, vol. 54, no. 6, pp. 2493-2507, Jun. 2008.

[12] O. Ozel, E. Ekrem, and S. Ulukus, "Gaussian wiretap channel with an amplitude constraint," in IEEE Inf. Theory Workshop in Lausanne, Switzerland, Sep. 2012, pp. 5553-5563.

[13] Z. Rezki and M. Alouini, "Secret-key agreement with public discussion over multi-antenna transmitters with amplitude constraints," in 2017 IEEE ISIT, Jun. 2017, pp. 1534-1538.

[14] P. T. Boggs and J. W. Tolle, "Sequential quadratic programming," Acta Numerica, vol. 4, p. 151, 1995. 